\title{
Characteristics of Liquid Marbles Formed with Plasma-Treated Hydrophobic Cellulose Powder
}

\author{
Tatsuhiko Ihara and Yu Iriyama* \\ Department of Biotechnology and Chemistry \\ Kinki University \\ 1 Umenobe, Takaya, Higashi-Hiroshima 739-2116, Japan \\ *Division of Interdisciplinary Sciences, \\ University of Yamanashi \\ 4-4-37 Takeda, Kofu, 400-8510 Japan \\ ihara@hiro.kindai.ac.jp
}

\begin{abstract}
Liquid marbles, non-stick droplets encapsulated by fine particles, were prepared with cellulose powders (CPs). Since CPs were very hydrophilic, they were treated with $\mathrm{O}_{2}$ plasma, followed by the adsorption of tetramethylcyclotetrasiloxane (TMCTS) before liquid-marble formation. Plasma treatment of CPs was carried out in a flask-rotation type reactor, which required $45 \mathrm{~min}$ to adsorb adequate amounts of TMCTS. In TMCTS adsorption, a successive reaction with ammonia was utilized for a secure chemisorption of TMCTS. After the adsorption, CPs became very hydrophobic. The prepared liquid marbles, water drops surrounded by TMCTS-adsorbed CP, were so robust that their compressibility exceeded $90 \%$. A liquid marble containing $0.1-\mathrm{M} \mathrm{CoCl}_{2}$ aq solution was applied for an ammonia gas sensor. In the presence of a highly diluted ammonia solution $(0.6 \%)$ in a desiccator, the color of the liquid marble turned from pink to blue within a few minutes.
\end{abstract}

Keywords: Liquid marble, Cellulose powder, Plasma treatment, Gas sensor, Tetramethylcyclotetrasiloxane

\section{Introduction}

Liquid marbles are defined as non-stick droplets encapsulated by micro- or nanometrically scaled particles. In 2001, Aussillous and Quéré [1] discovered that fluorinated silane-treated highly hydrophobic lycopodium grains could encapsulate a liquid droplet by self-organizing on the liquid-vapor interface and to prevent the liquid from contacting surfaces that it encountered. This is the origin of the name of "liquid marble", and unique dynamic behavior was first demonstrated. Since their discovery, interest in this liquid marbles has increased during the past decade, for both scientific and technological reasons. It has already been demonstrated a potential for various applications, such as gas sensing [2], ferrofluidic applications $[3,4]$, microreactors $[5,6]$ and microreservoirs [7]. As for the materials of hydrophobic powder is concerned, fluorinecontaining polymers such as polytetrafluoro- ethylene (PTFE), poly(vinylidene fluoride), poly(perfluoroalkyl acrylate) were used since a hydrophobic property was important to form liquid marbles. Liquid marbles are also found naturally; for example, galling aphids species convert honeydew droplets into marbles [8]. Aphids produce marbles with a secreted powdery wax, which has three distinct roles: (1) it is hydrophobic, (2) it creates a microscopically rough inner gall surface made of weakly compacted wax needles making the gall ultra-hydrophobic, and (3) it coats the honeydew droplets, converting them into liquid marbles that can be rapidly and efficiently moved [9]. Bisco et al. lead to the following formula for the apparent contact angle $\theta^{*}$ on a rough surface:

$$
\cos \theta^{*}=-1+\varphi(1+\cos \theta)
$$

where $\varphi$ is the areal fraction of liquid-solid contact and $\theta$ is the contact angle on a smooth substrate of the same material. If $\varphi<<1$ and the substrate is hydrophobic $\left(\theta>90^{\circ}\right)$, the effect of roughness is to increase the contact angle. 
In this research, we examined the preparation of hydrophobic cellulose powder (CP) in order to fabricate robust liquid marbles. Strong network interaction between CPs with ultrahydrophobicity could be expected at the self-organization since cellulose is a fiber material with rough surface. In order to give a hydrophobic property on CP surface, we applied gas-phase successive adsorption method using cyclosiloxane and ammonia following oxygen plasma treatment.

\section{Experimental}

\subsection{Materials}

Cellulose powders (CPs) of three different particle sizes manufactured by Nippon Paper Chemicals were used in this work: they are W-50 (50 mesh powder, passed $>90 \%$ ); W-300G (300 mesh powder, passed $>90 \%$ ); and $\mathrm{W}-10 \mathrm{MG}$. They are all water insoluble natural CP and the first two CPs are food additive grade with a brand name of KC Flock and the last one is food material grade with a brand name of NP Fiber. Their average particle diameter and specific surface area are shown in Table 1. The particle size was estimated by the observation of SEM, and specific surface area was measured by Brunauer-Emmett-Teller (BET) method. They are called "powder", but actually they are fiber-form structure. Therefore, there seems to be not clear correlation between diameter and surface area.

1,3,5,7-Tetramethylcyclotetrasiloxane (TMCT S) supplied from Shin-Etsu Chemical Co. was used without further purification. Ammonia aq solution was $28 \%$ special grade from Kanto Chemical Co., which was used for TMCTS as received and used for ammonia sensor after 50-times dilution.

Table 1. Properties of CPs used in this study.

\begin{tabular}{ccc}
\hline & $\begin{array}{c}\text { Particle diameter } \\
(\mu \mathrm{m})\end{array}$ & $\begin{array}{c}\text { Specific surface } \\
\text { area }\left(\mathrm{m}^{2} / \mathrm{g}\right)\end{array}$ \\
\hline W-50 & 45 & 1.48 \\
W-300G & 28 & 0.73 \\
W-10MG2 & 10 & 2.04 \\
\hline
\end{tabular}

\subsection{Oxygen Plasma Treatment}

The oxygen plasma treatment of $\mathrm{CP}$ was carried out similarly as previous report [10] in which the rotatable-reactor type shown in Figure 1 was used. The reactor, designed for powder treatment, is equipped with a $1000-\mathrm{mL}$ flask which is kept rotating during the treatment for a uniform treatment. Twenty grams per one batch $\mathrm{CP}$ was introduced into the reactor, and then oxygen plasma treatment was carried out at a fixed oxygen flow late, pressure and rf power of $5 \mathrm{sccm}, 40 \mathrm{~Pa}$ and $45 \mathrm{~W}$, respectively for given periods of time. Oxygen flow rate and rf power was controlled by mass flow controller (STEC) and rf (13.56 MHz) generator (ADTEC, AX-1000).

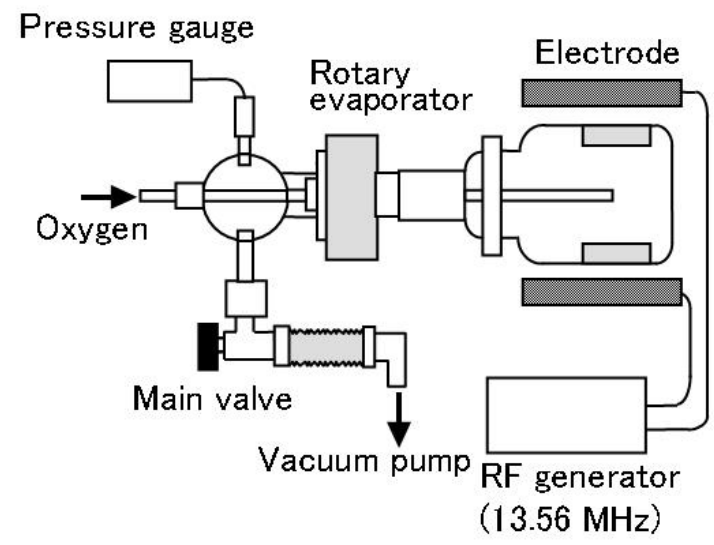

Figure 1. Plasma apparatus for CPs.

\subsection{Adsorption of TMCTS}

In the first step of the adsorption, a weighing bottle containing $5 \mathrm{~mL}$ of TMCTS was placed in the bottom stage of a vacuum desiccator of 19-L capacity, and 20 Petri dishes containing $1 \mathrm{~g}$ of uniformly spread plasma-treated $\mathrm{CP}$ were put on the middle stage of the desiccator. The desiccator was evacuated down to $5.3 \times 10^{4} \mathrm{~Pa}$ and kept for 12 h. Then, physisorbed TMCTS were removed under vacuum below $6.7 \mathrm{~Pa}$ at $50{ }^{\circ} \mathrm{C}$ for $2 \mathrm{~h}$. In the second step, the CP with chemisorbed TMCTS was replaced to another desiccator with ammonia atmosphere and kept for $1 \mathrm{~h}$ for the hydrolysis of chemisorbed TMCTS. Then, physisorbed ammonia was removed under vacuum with below $6.7 \mathrm{~Pa}$ at $50{ }^{\circ} \mathrm{C}$ for $2 \mathrm{~h}$. These sequences were repeated up to three times.

\subsection{Formation of Liquid Marble}

Formation of liquid marble was performed using clock glass setup method which McEleney et. al. [11] recommended. This method consist of five steps as follows: (a) several grams of ТPCP were spread in clock glass, (b) water drop is placed on the powder surface, (c) clock glass is agitated and tilted to allow powder to fully cover water surface, (d) the liquid marble is then rolled towards the edge, and (e) finally exits the setup. Obtained liquid marbles were put into following experiments for the investigation of physical properties and availability for ammonia gas sensor was examined according to Tian's report [2]. All experiments 
were carried out at $22{ }^{\circ} \mathrm{C}$. Figure 2 shows photographs for the formation process of liquid marble.
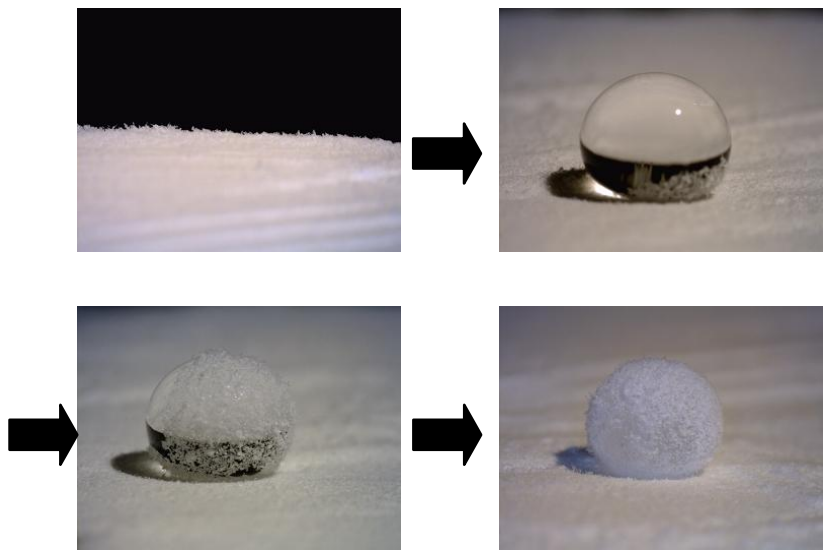

Figure 2. Formation of liquid marble.

\subsection{Measurements}

\subsubsection{Digital Microscopy}

Marble specimens were examined under microscope using a Dino-Lite digital microscope and DinoCapture 2.0 software.

\subsubsection{Hydrophobicity}

$0.2 \mathrm{~g}$ of CP sample was taken into Erlenmeyer flask with $50 \mathrm{~mL}$ of ion exchanged water. Then methanol was added from buret until $\mathrm{CP}$ was completely dispersed under magnetically stirring. The hydrophobicity (\%) was obtained by following equation: hydrophobicity $(\%)=$ amount of methanol added $(\mathrm{mL}) \times 100 /$ (amount of methanol added $(\mathrm{mL})+$ amount of ion exchange water $(\mathrm{mL}))$.

\subsubsection{Contact Angle}

Contact angle of water for TPCPs was measured by contact angle measurement apparatus equipped with Dino-Lite digital microscope and image-processing software (Motic Image Plus 2.2S, Motic). TPCP samples for this measurement were fabricated to tablet $10 \mathrm{~mm}$ in diameter using pellet molding machine. A water droplet of $10 \mu \mathrm{L}$ was put onto the pellet surface, and contact angle was measured from the picture of the side view.

\subsubsection{Dispersibility in Water}

$0.2 \mathrm{~g}$ of $\mathrm{CP}$ and $50 \mathrm{~mL}$ of ion exchanged water are charged in a 100-mL color comparison tube, and agitated vigorously. The dispersibility of CPs was evaluated by the observation of the dispersion state $24 \mathrm{~h}$ after the agitation.

\subsubsection{Compressibility of Liquid Marbles}

In order to measure the robustness of liquid marbles formed with $\mathrm{CP}$, we measured the compressibility using outside micrometer (Mitsutoyo, M300) with a flat tip. The initial diameter $d_{0}$ of the liquid marble before compression and the diameter $d_{\mathrm{d}}$ when it destroyed by compression were measured, and compressibility $(\%)$ was obtained from $\left(\left(d_{0}-d_{\mathrm{d}}\right) / d_{0}\right)$ $\times 100$. Amount of water for a water droplet was set to $15 \mu \mathrm{L}$, of which $d_{0}$ for liquid marble was 3.2 $\mathrm{mm}$.

\subsubsection{Application for Gas Sensor}

Ammonia-sensing ability was investigated in a closed system with $\mathrm{NH}_{3}$ aq solution. Namely, the weighing bottle containing $5 \mathrm{~mL}$ of 50 -times diluted $\mathrm{NH}_{3}$ aq solution $(0.6 \%)$ was placed on the bottom stage of a desiccator (19 L), and Petri dish in which a liquid marble loaded with indicator was placed on the middle stage of the desiccator. For the indicator, $0.1-\mathrm{M} \mathrm{CoCl}_{2}$ solutions were used. In the reaction with $\mathrm{NH}_{3}$, the color of $\mathrm{CoCl}_{2}$ turns blue. The amount of indicator solution was set to $15 \mu \mathrm{L}$.

\section{Results and Discussion}

\subsection{Adsorption of TMCTS}

Figure 3 shows the relationship between adsorbed amounts of TMCTS and $\mathrm{O}_{2}$ plasma treatment time for the three kinds of CPs. The number of the adsorption cycle is 1 . Usually, a fairly long time is required for the treatment of powders, but in this case of $\mathrm{CP}$ treatment, as seen

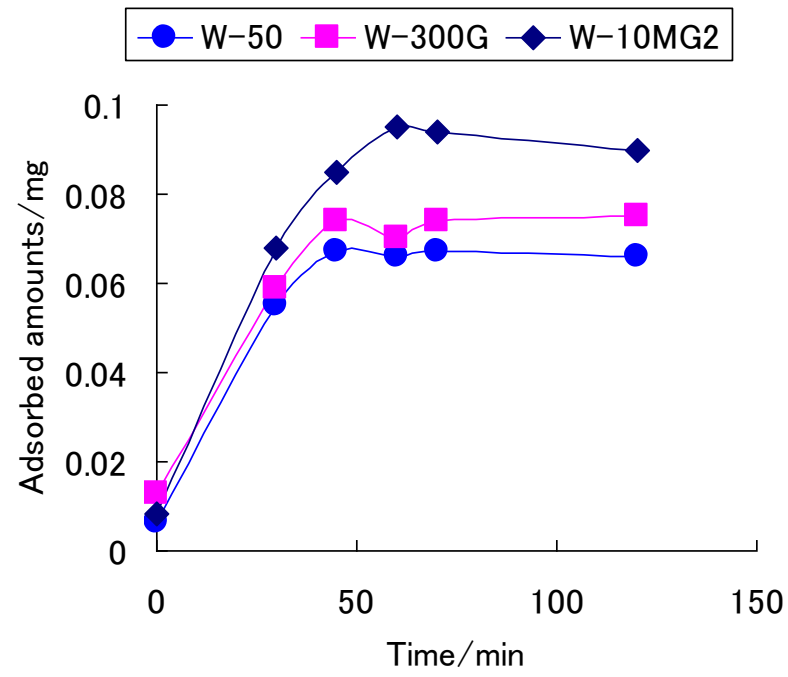

Figure 3. Relationship between $\mathrm{O}_{2}$ plasma treatment time and adsorbed amounts of TMCTS per $1 \mathrm{~g} \mathrm{CP}$. 
in the Figure, 45 min of treatment time was found to be adequate. Therefore, we fixed the treatment time to be $45 \mathrm{~min}$ in the rest of the experiment. In the comparison among the three CPs, the order of the adsorption amounts is as follows: $\mathrm{W}-10 \mathrm{MG} 2>$ $\mathrm{W}-300 \mathrm{G}>\mathrm{W}-50$, which is inverse tendency against the particle size. We used $\mathrm{O}_{2}$ plasma for the expectation of the formation of carboxy group, which reacts with hydrosilyl $(\mathrm{Si}-\mathrm{H})$ group of TMCTS.

\subsection{Hydrophobicity of Plasma-Treated and} TMCTS-adsorbed CPs

The surface of CPs became very hydrophobic after TMCTS adsorption. Figure 4 shows the relationship between hydrophobicity of CPs and the number of adsorption cycle of TMCTS for the three kinds of CPs. The hydrophobicity was measured as described in Experimental 2.5.2. Because untreated CPs are very hydrophilic, their hydrophobicity is zero, which is not shown in the Figure, but as a comparison, untreated and 3-cycle adsorbed CPs are shown.

In all the three CPs, the hydrophobicity increased with the increasing number of adsorption cycle. The hydrophobicity of even three-cycleadsorbed untreated $\mathrm{CP}$ was lower than that of one-cycle-adsorbed plasma-treated one for each corresponding CP. Therefore, plasma treatment before TMCTS adsorption is proved to be very effective. Among the three CPs, the hydrophobicity increased in the following order: $\mathrm{W}-300 \mathrm{G}<\mathrm{W}-10 \mathrm{MG} 2<\mathrm{W}-50$. This tendency is different from that of adsorption amounts of TMCTS. One of the reasons may be that the CPs

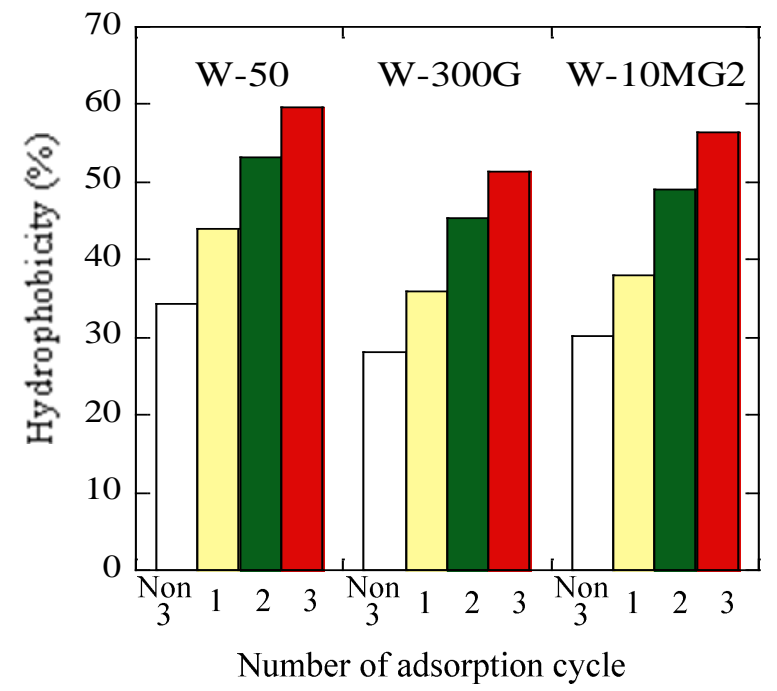

Figure 4. Relationship between hydrophobicity of CPs and the number of adsorption cycle of TMCTS for the three kinds of CPs. "Non" denotes "Non-plasma treatement"
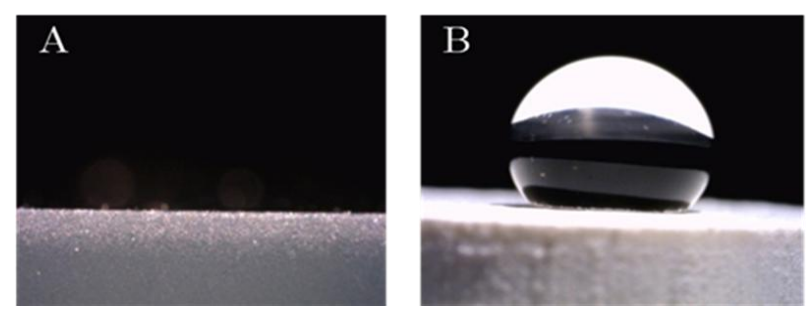

Figure 5. Photographs of water droplet placed on untreated (A) and plasma-treated and 3-times TMCTS-adsorbed (B) W-50 pellets.

are fiber-form, and effective hydrophobicity is thought to be obtained by not only adsorbed amounts but also adsorbed density of TMCTS.

The hydrophobicity can be also estimated by the measurement of contact angle. Figure 5 shows that the appearance of water drop on untreated W-50 and plasma-treated and 3-cycle TMCTS-adsorbed W-50, both of which were press-formed to be pellet. In untreated CP pellet, a water droplet was absorbed immediately and contact angle could not be measured as shown in Fig.5A, while TMCTS-adsorbed CP was very hydrophobic and showed the contact angle as high as $130^{\circ}$ as shown in Fig. $5 \mathrm{~B}$ which was taken 10 min after water-drop placement.

\subsection{Dispersibility of TMCTS-Adsorbed CPs}

Figure 6 (1)-(6) is the photograph of the

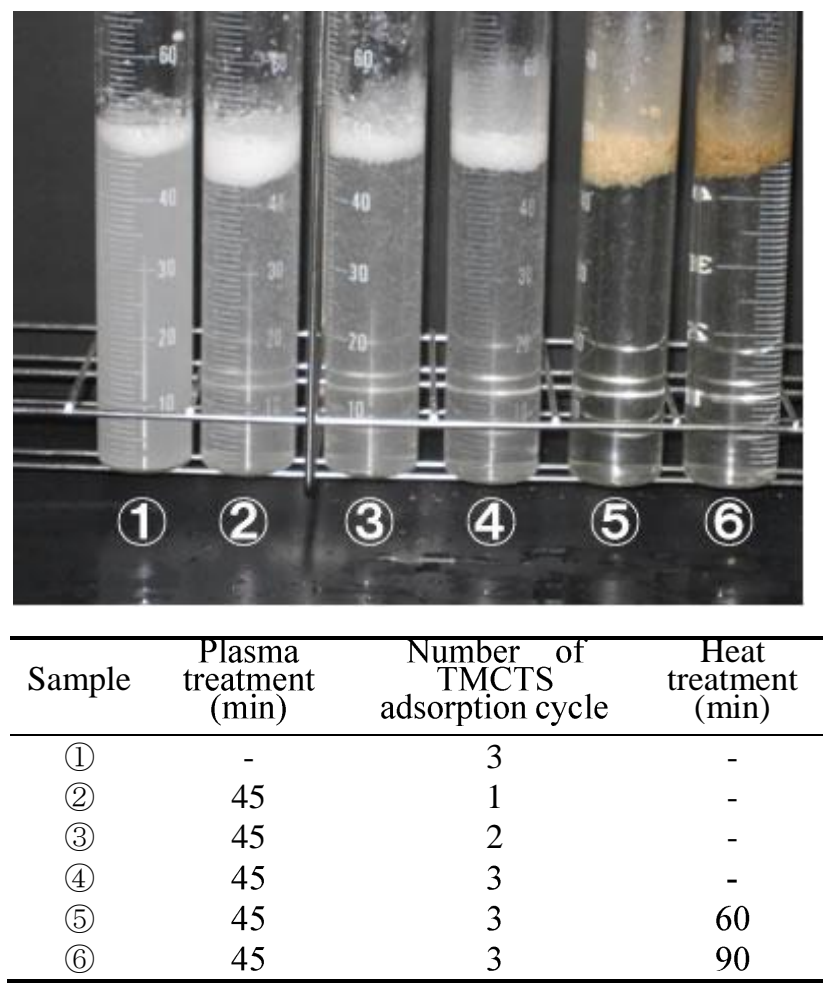

Figure 6. Dispersion states of TMCTS-adsorbed W-50 in water. 
dispersion of TMCTS-adsorbed untreated (3 cycles) and plasma-treated (1-3 cycles) $\mathrm{W}-50$ in water. Twenty-four hours after vigorous shaking, untreated W-50 (1) is still being dispersed appreciably although it was proceeded three-cycle TMCTS adsorption. For the plasma-treated W-50, on the other hand, the dispersibility became worse as the number of the TMCTS-adsorption cycle increased ( (2) - (4) ). However, even for the three-cycle TMCTS-adsorbed W-50(4)), a little turbidity is observed.

This phenomenon is thought that TMCTS adsorbed on CP is not hydrophobic enough probably because not all four hydrosilyl (Si-H) groups of the cyclic siloxane are bonded on $\mathrm{CP}$ after hydrolysis. Some of them seem to remain unreacted and some other TMCTS stay as silanols $(\mathrm{Si}-\mathrm{OH})$ which exhibit hydrophilic property. Therefore, in order to reduce the silanol OH groups, we heat treated TMCTS-adsorbed CP. The hydrophobicity is expected to increase by the heat treatment if all the silanols react with $\mathrm{CP}$ or dehydrate themselves. The condition of the heat treatment was $200{ }^{\circ} \mathrm{C}$ for 60 and 90 min. Fig. 6 (5) and (6) are the dispersion of 60- and 90-min heat-treated and TMCTS-adsorbed W-50. The color of the CP became brown as the heat-treatment time increased, and the dispersibility became worse, which implies that more hydrophobicity was achieved.

The hydrophobicity of heat-treated TMCTSadsorbed $\mathrm{W}-50$ is shown in Table 2. The heat-treated CP showed higher hydrophobicity, and longer heat treatment time was found to be effective.

Table 2. Hydrophobicity of heat-treated W-50.

\begin{tabular}{ccc}
\hline $\begin{array}{l}\text { Sample \# } \\
\text { in Fig.6 }\end{array}$ & $\begin{array}{c}\text { Heat treatment } \\
(\mathrm{min})\end{array}$ & $\begin{array}{c}\text { Hydrophobicity } \\
(\%)\end{array}$ \\
\hline$(4)$ & 0 & 61.0 \\
$(5)$ & 60 & 61.8 \\
(6) & 90 & 64.2 \\
\hline
\end{tabular}

Plasma treatment, 45 min; TMCTS adsorption cycle, 3.

\subsection{Compressibility of Liquid Marble}

Compressibility of liquid marbles prepared with TMCTS-adsorbed $\mathrm{CP}$ was measured. The results are shown in Figure 7, in which 3-cycle TMCTS-adsorbed CPs were compared. Before heat treatment, in this Figure, W-50 showed the best compressibility (93.9\%) among the three CPs. After heat treatment, in addition, the compressibility of W-50 further increased to more than $95 \%$, and $60-\mathrm{min}$ heat treated $\mathrm{CP}$ was the best. Figure 8 is a photograph of the compression test.

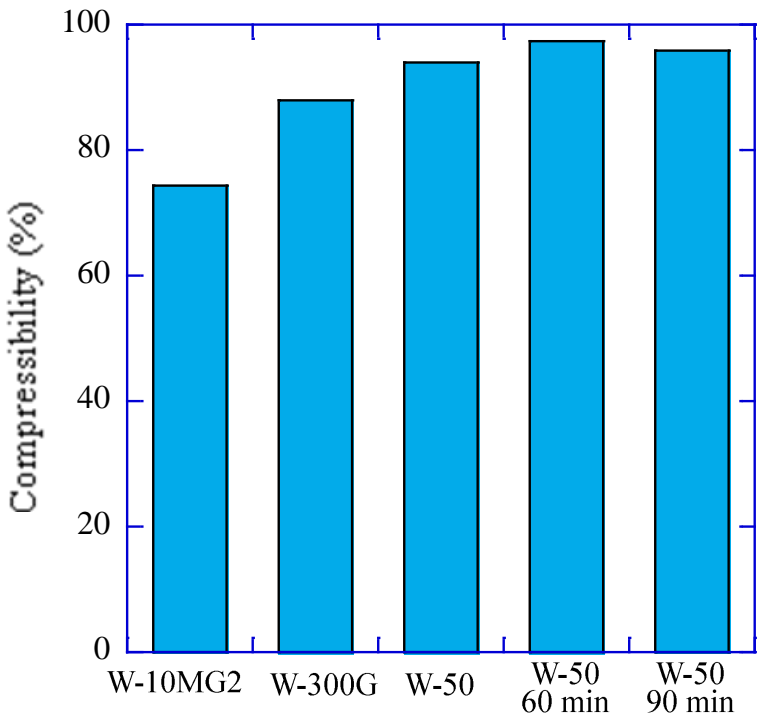

Figure 7. Compressibility of liquid marbles prepared with TMCTS-adsorbed CPs (W-10MG2, W-300G, W-50) and heat-treated W-50. "60 min" and "90 min" are heat treatment time. TMCTS adsorption cycle, 3 .

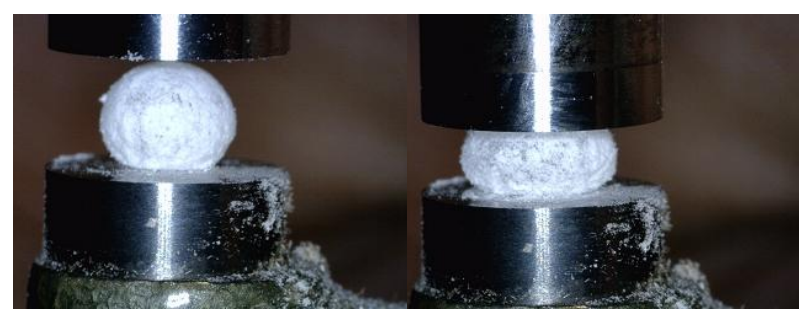

(A)

(B)

Figure 8. Compression test of CP.

3.6. Application of Liquid Marble to Ammonia Sensor

Liquid marble is covered with the network of hydrophobic powders. Therefore, gases can pass through the network. Utilizing this unique feature, liquid marble is applied to gas sensor.

Figure 9 shows the photograph of $\mathrm{CoCl}_{2}$ containing liquid marble formed with TMCTS-adsorbed W-300G used for ammonia gas sensor: (A, pink) before and (B, blue) after contact with ammonia. The concentration of used ammonia was 50-times diluted ammonia solution $(0.6 \%)$, which is almost impossible for human's nose to detect. The color change of the liquid marbles started just about $1 \mathrm{~min}$ after the contact, and then the color became clear and stable blue about after another $4 \mathrm{~min}$. 


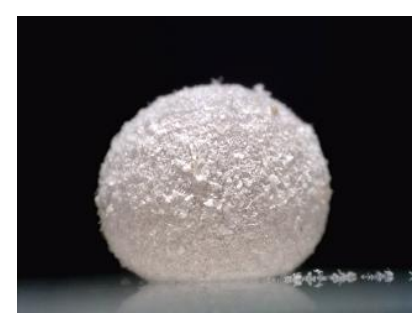

(A)

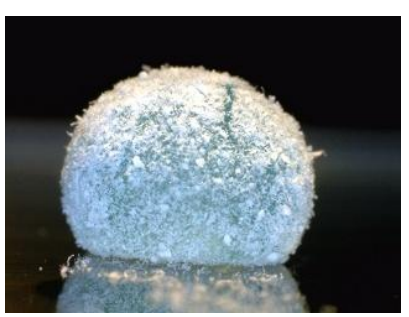

(B)
Figure 9. Photographs of $\mathrm{CoCl}_{2}$ containing liquid marbles formed with TMCTS-adsorbed W-300G (A, pink) before and (B, blue) after contact with ammonia..

\section{Conclusion}

Three kinds of CPs (W-50, W-300G, $\mathrm{W}-10 \mathrm{MG} 2$ ) were used for the preparation of liquid marbles after $\mathrm{O}_{2}$ plasma treatment and TMCTS adsorption. The adsorbed amounts of TMCTS increased with increasing number of the successive adsorption cycle. W-10MG2 (smallest diameter) showed the highest adsorption amounts, while W-50 (largest diameter) exhibited the highest hydrophobicity and compressibility. Since gases can pass through the $\mathrm{CP}$ on the surface of liquid marbles, a liquid marble containing $\mathrm{CoCl}_{2}$ was found to be used as a very sensitive ammonia sensor, in which the color of the liquid marbles turned from pink to blue.

\section{References}

1. P. Aussillous and D. Quéré, Nature, 411, 924 (2001),

2. J. Tian, T. Arbatan, X. Li, and W. Shen, Chem. Commun., 46, 4734 (2010).

3. E. Bormashenko, R. Pogreb, Ye. Bormashenko, A. Musin, and T. Stein, Langmuire, 24, 12119 (2008).

4. Y. Zhao, J. Fang, H. Wang, and T. Lin, $A d v$. Mater., 22, 707 (2010)

5. L. Gao and T. J. MacCarthy, Langmuir, 23, 10445 (2007).

6. N. Eshtiaghi, J. J. S. Liu, and K. P. Hapgood, Powder Technol., 197, 184 (2010).

7. W. Wang, L. C. Bray, D. J. Adams, A. I. Cooper, J. Am. Chem. Soc., 130, 11608 (2008).

8. N. Pike, D. Richard, W. Foster, and L. Mahadevan, Proc. R. Soc. London, Ser. B, 265, 1211 (2002).

9. J. Bisco, C. Marzolin, and D. Quéré, Europhys. Lett., 47, 220 (1999).

10. Y. Iriyama, K. Ohbayashi, and T. Ihara, $J$. Photopolym. Sci. Technol., 19, 215 (2006).

11. P. McEleney, G. M. Walker, I. A. Larmour, and S. E. J. Bell, Chem. Eng. J., 147, 373 (2009) 